\title{
具有新颖金属味唑构筑单元的金属有机框架材料合成 与性能
}

\author{
唐玉环 ${ }^{1,2}$, 王飞 ${ }^{1 *}$, 张健 ${ }^{1 *}$ \\ 1. 中国科学院福建物质结构研究所, 结构化学国家重点实验室, 福州 350002; \\ 2. 中国科学院大学, 北京 100049 \\ *联系人, E-mail: wangfei04@fjirsm.ac.cn; zhj@fjirsm.ac.cn \\ 2018-01-15 收稿, 2018-02-27 修回, 2018-03-05 接受, 2018-04-16 网络版发表 \\ 国家自然科学基金 $(21425102,21573236)$ 资助
}

\begin{abstract}
摘要利用咪唑衍生物2-(1-羟基-乙基)-3氢-苯并咪唑-5-羧酸(Hebimc)和金属锌盐反应得到一种新颖的次级构 筑单元 $\mathrm{Zn}_{2}(\mathrm{ebimc})_{2}$. 在此基础上，通过加入辅助配体和条件优化，成功合成了4例结构新颖的金属有机框架 (MOFs) 材料, 其分子式分别为: $\mathrm{Zn}_{2}(\mathrm{Hebimc})_{4}(\mathbf{1}), \mathrm{Zn}_{2}(\mathrm{Hebimc})_{2}(\mathrm{BDC}) \cdot x \mathrm{G}\left(\mathbf{2}, \mathrm{BDC}=\right.$ 对苯二甲酸, $\mathrm{G}=$ 客体分子), $\mathrm{Zn}_{2}$ $(\text { Hebimc })_{2}(\mathrm{NDC}) \cdot x \mathrm{G}(\mathbf{3}, \mathrm{NDC}=2,6$ - 荟二酸 $), \mathrm{Zn}_{8}(\mathrm{ebimc})_{6}(\mathrm{DOBDC})(\mathrm{OH})_{2}\left(\mathrm{H}_{2} \mathrm{O}\right)_{2} \cdot x \mathrm{G}(\mathbf{4}, \mathrm{DOBDC}=2,5-$ 二着基对苯二甲 酸)。其中, 化合物 $\mathbf{1} 由 \mathrm{Zn}_{2}(\text { Hebimc })_{2}$ 单元自身首尾相连形成一维链结构; 在化合物 $\mathbf{2}$ 和 $\mathbf{3}$ 中, 通过加入辅助羧酸配体 BDC或NDC, $\mathrm{Zn}_{2}(\text { Hebimc })_{2}$ 单元被辅助配体连接形成二维层状结构; 在化合物 $\mathbf{4}$ 中, 有机碱的加入使得Hebimc配体 上的羒基和咪唑基同时脱去质子, 表现出高的配位能力, $\mathrm{Zn}_{2}(\mathrm{Hebimc})_{2}$ 单元和单核 $\mathrm{Zn}$ 相互连接形成层状结构, 再通 过DOBDC配体链接形成柱层式的三维框架. 有意思的是, 利用化合物 $\mathbf{4}$ 中的羟基官能团-OH和孔道限域效应, 可 以还原 $\mathrm{Ag}^{+}$离子形成均匀分散的纳米粒子, 得到的复合材料 $\mathrm{Ag} @ \mathbf{4}$ 对结晶紫水溶液有较好的光催化降解效果. 本文 对于金属有机框架材料的结构调控提供了新思路.
\end{abstract}

关键词金属-有机框架材料, 晶体工程, 可控合成, 单晶结构, 染料降解

金属有机骨架(MOFs)材料是一类由金属离子或 者簇单元通过有机配体连接形成的具有周期性网络 结构的晶态多孔材料. 金属有机框架材料具有比表 面积大、孔道可调控性、结构丰富以及可以定向设计 等特点, 在气体吸附与存储、催化、离子交换与分离、 化学传感等多个领域具有潜在的应用前景 ${ }^{[1 \sim 4]}$. 其中, 用有机配体连接次级构筑单元 (SBUs) 是合成新型 MOFs材料以及拓展材料性能的一种非常有效的方 法 $^{[5 \sim 18]}$. 很多知名 MOFs材料具有典型的次级构筑单 元, 比如, HKUST-1 具有 $\left[\mathrm{Cu}_{2}(\mathrm{COO})_{4}\right]$ 单元 ${ }^{[9]}$, MIL101 具有 $\left[\mathrm{M}_{3}(\mathrm{COO})_{6}\right]$ 单元 ${ }^{[10]}, \mathrm{MOF}-5$ 和 MOF- 177 具
有 $\left[\mathrm{Zn}_{4} \mathrm{O}(\mathrm{COO})_{6}\right]$ 单元 ${ }^{[11]}, \mathrm{UiO}-66$ 具有 $\left[\mathrm{Zr}_{6} \mathrm{O}\left(\mu_{3}-\mathrm{O}\right)_{4}\right.$ $\left.\left(\mu_{3}-\mathrm{OH}\right)_{4}(\mathrm{COO})_{12}\right]$ 单元 ${ }^{[12]}$ 等. 近年来, 科学家们提出 了网络化学的方法(isoreticular chemistry ${ }^{[13]}$ : 基于同 一种次级构筑单元和框架网络, 利用不同尺寸长度 和修饰基团的同类型配体，可以有效调控目标材料 的各种性能. 因此, 开发新的次级构筑单元对 MOFs 材料的合成有着重要的意义. 如上所述, 大部分的次 级构筑单元是由羧酸配体和金属中心原位反应而形 成的. 除了羧酸，一些含有氮杂环的氮唑类有机配体 也是合成MOFs材料的常用配体，比如咪唑、三氮唑、 四氮唑等 ${ }^{[14,15]}$. 相对来讲, 基于氮唑类的次级构筑单 
元要少得多, 可能是氮杂环比较刚性, 位阻比羧基 大, 不太容易形成次级构筑单元. 该类型配体更倾向 于形成基于单核金属中心的拓展结构, 比如基于咪 唑类配体的沸石咪唑框架材料(ZIFs). 因此, 选择带 有合适官能团的有机配体是合成新颖次级构筑单元 的关键.

咪唑衍生物2-(1-差基-乙基)-3氢-苯并咪唑-5-羧 酸 (Hebimc) 既有羧基又包含咪唑环, 同时还有含羟 基的手性中心, 是一种多功能咪唑类配体: (1) 羧基 可以与金属中心配位形成次级构筑单元; (2) 咪唑基 团可以提供 $\mu_{2}$-桥联配位模式形成ZIFs材料; (3) 羟基 增强目标材料和客体分子的相互作用; (4) 采用单手 性的配体有望合成出单手性的MOFs材料, 拓展其在 手性相关的应用. 尽管该配体合成方法简单, 反应原 料也很便宜, 但是用它合成MOFs材料的报道却很 少 ${ }^{[19 \sim 21]}$.

本研究利用Hebimc作为有机配体和金属锌盐反 应. 研究结果发现, Hebimc配体并没有和金属中心形 成类似ZIFs的单核结构，也没有形成基于羧基的次 级构筑单元, 而是以鳌合配位的模式和金属中心形 成一个罕见的 $\mathrm{Zn}_{2}(\mathrm{Hebimc})_{2}$ 单元(图 1). 基于此单
元，通过添加羧酸作为辅助配体以及对合成条件 进行优化, 成功实现了从一维(1D)到二维(2D)再 到三维(3D)MOFs材料的可调合成. 下文将详细 介绍这些化合物.

\section{1 实验}

( i ) 仪器与试剂. 本文所用测试仪器包括 Rigaku Saturn 724+小分子衍射仪，XcaliburE小分子 衍射仪, Mo $K \alpha(\lambda=0.71073 \AA$ 光源辐射; Rigaku Mercury70小分子衍射仪, Mo $K \alpha(\lambda=0.71073 \AA$ 光源 辐射; STA449F3综合热分析仪, 测试环境为氮气气 氛, 升温速率为 $10^{\circ} \mathrm{C} / \mathrm{min}$; MiniFlex-II X射线粉末衍射 仪, $\mathrm{Cu} \mathrm{K \alpha}(\lambda=1.5418 \AA)$ 光源辐射, 扫描速度 $5 \% \mathrm{~min}$; Lambda350Magna紫外仪; 750傅里叶变换红外光谱仪 $\left(500 \sim 4000 \mathrm{~cm}^{-1}\right)$, 采用 $\mathrm{KBr}$ 压片测试. 试剂均是商业 购买, 未进行进一步纯化.

(ii ) Hebimc配体的合成. 将3,4-二氨基苯甲酸 (12.16 g, $80 \mathrm{mmol})$, 乳酸 $(5 \mathrm{~mL}, 200 \mathrm{mmol}$ )和盐酸 (4 $\mathrm{mol} / \mathrm{L}, 100 \mathrm{~mL}$ )搅拌约 $5 \mathrm{~min}$, 然后在 $120^{\circ} \mathrm{C}$ 的油浴中 回流并加热 6 h. 将沉淀滤出后，用氢氧化钠水溶液 $(1 \mathrm{~mol} / \mathrm{L})$ 调节 $\mathrm{pH}$ 至 $8 \sim 9$, 将得到的沉淀滤出，用去离

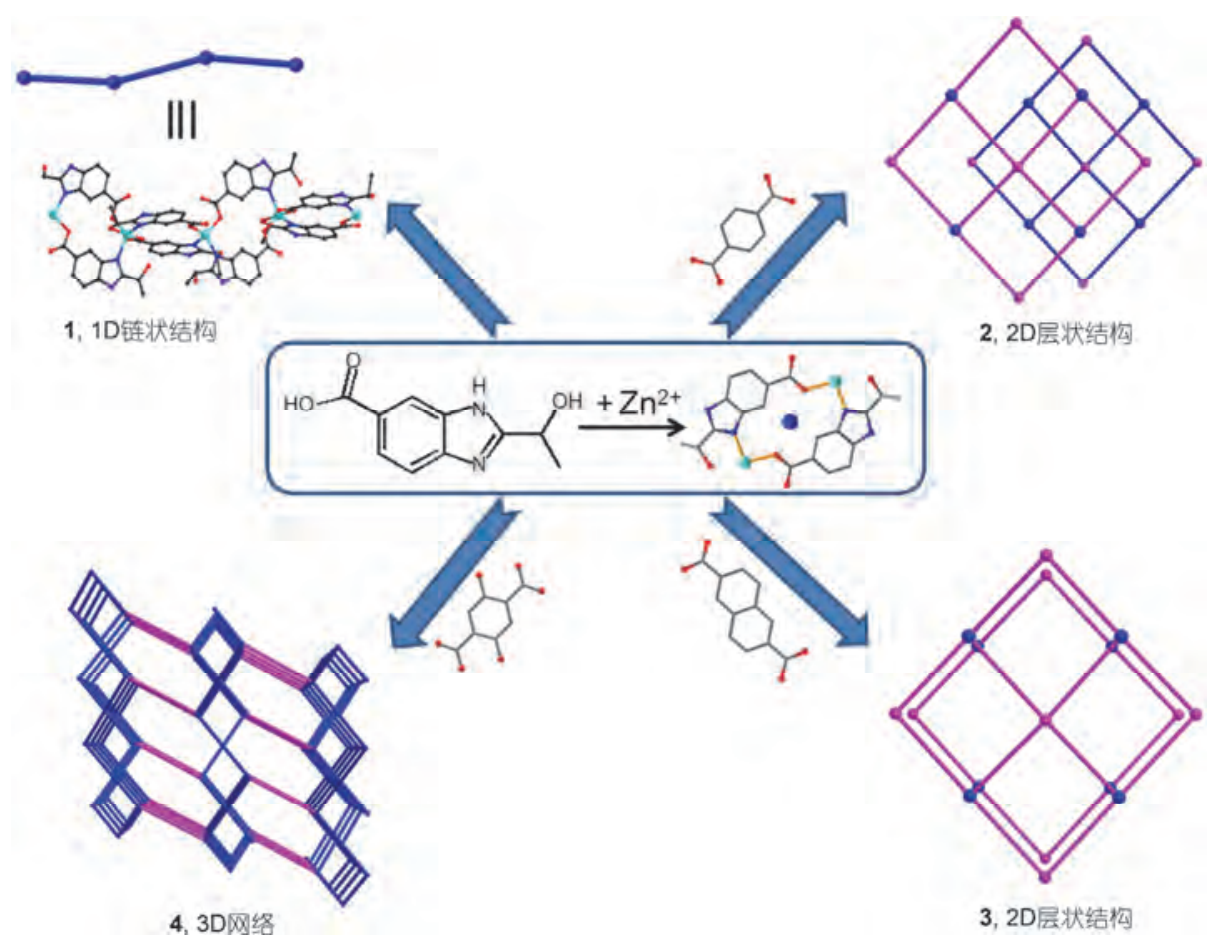

图 1 (网络版彩色)化合物 1 4 的调控合成示意图. 在不同的合成条件下得到具有 1D到 2D再到 3D的丰富拓扑结构的化合物

Figure 1 (Color online) The controllable synthesis of compounds 1-4. Different synthetic conditions result into 1D chains in 1, sql topology in 2 and $\mathbf{3}$, and the piller-layer framework in $\mathbf{4}$ 
子水洗涤并在空气中干燥后得到晶体样品 $(7.3 \mathrm{~g}$, 产 率: 35\%, 基于基于3,4-二氨基苯甲酸).

( iii ) 化合物 $\mathbf{1}$ 的合成. 将 $\mathrm{ZnSiF}_{6} \cdot 6 \mathrm{H}_{2} \mathrm{O}(0.2$ $\mathrm{mmol}, 0.0620 \mathrm{~g})$, Hebimc配体 $(0.1 \mathrm{mmol}, 0.0209 \mathrm{~g}$ )和3 $\mathrm{mL}$ 水混合均匀, 放人 $100{ }^{\circ} \mathrm{C}$ 的恒温烘箱中加热 $3 \mathrm{~d}$, 有浅棕色的块状晶体产生; 冷却至室温并过滤, 用乙 醇洗涤, 得到干燥的晶体样品(产率: 40\%, 基于配体 Hebimc).

(iv) 化合物 2 的合成. 将 $\mathrm{ZnSiF}_{6} \cdot 6 \mathrm{H}_{2} \mathrm{O}(0.1$ $\mathrm{mmol}, 0.0310 \mathrm{~g})$, 对苯二甲酸 $(\mathrm{BDC})$ 配体 $(0.1 \mathrm{mmol}$, $0.016 \mathrm{~g})$ 和Hebimc配体 $(0.1 \mathrm{mmol}, 0.022 \mathrm{~g})$, 以及 $6 \mathrm{~mL}$ 异丙醇和 $3 \mathrm{~mL}$ 水混合均匀, $100^{\circ} \mathrm{C}$ 的恒温烘箱中加热 $3 \mathrm{~d}$, 有浅棕色的块状晶体产生; 冷却至室温并过滤, 然后用乙醇洗涤, 得到干燥的晶体样品(产率: $65 \%$, 基于配体Hebimc).

( V ) 化合物 3 的合成. 将 $\mathrm{Zn}\left(\mathrm{NO}_{3}\right)_{2} \cdot 6 \mathrm{H}_{2} \mathrm{O}(0.5$ $\mathrm{mmol}, 0.150 \mathrm{~g})$, 吡嗪 $(0.5 \mathrm{mmol}, 0.040 \mathrm{~g})$, 四乙基溴 化铵 (TEAB, $0.3 \mathrm{mmol}, 0.063 \mathrm{~g}), \mathrm{NDC}$ 配体 $(0.3$ mmol, $0.035 \mathrm{~g})$, Hebimc配体 $(0.3 \mathrm{mmol}, 0.067 \mathrm{~g}), 3 \mathrm{~mL}$ $\mathrm{N}, \mathrm{N}$-二甲基甲酰胺(DMF) 和 $1.5 \mathrm{~mL}$ 水混合均匀, 放人 $120^{\circ} \mathrm{C}$ 恒温烘箱中加热 $3 \mathrm{~d}$, 有浅棕色的块状晶体产 生; 冷却至室温并过滤, 然后用乙醇洗涤, 得到干燥 的晶体样品(产率: 78\%, 基于配体Hebimc).

( vi ) 化合物 4 的合成. 将 $\mathrm{Zn}\left(\mathrm{NO}_{3}\right)_{2} \cdot 6 \mathrm{H}_{2} \mathrm{O}(0.5$ $\mathrm{mmol}, 0.150 \mathrm{~g})$, 吡嗪 $(0.5 \mathrm{mmol}, 0.040 \mathrm{~g})$, 三苯基膦 (0.3 mmol, $0.079 \mathrm{~g})$, DOBDC配体 $(0.3 \mathrm{mmol}, 0.059 \mathrm{~g})$ 和Hebimc配体 $(0.3 \mathrm{mmol}, 0.067 \mathrm{~g})$ 溶于 $6 \mathrm{~mL}$ DMF和 $1.5 \mathrm{~mL}$ 水中, 放人 $120^{\circ} \mathrm{C}$ 恒温烘箱中 $3 \mathrm{~d}$, 有浅黄色的 块状晶体产生; 冷却至室温并过滤, 然后用乙醇洗 涤, 得到干燥的晶体样品 (产率: 50\%, 基于配体 Hebimc).

(vii) $\mathrm{X}$ 射线晶体结构测定. 选择晶体大小为 $0.15 \mathrm{~mm} \times 0.10 \mathrm{~mm} \times 0.05 \mathrm{~mm}$ 的化合物 $\mathbf{1}, 0.10 \mathrm{~mm} \times$ $0.05 \mathrm{~mm} \times 0.05 \mathrm{~mm}$ 的化合物 $\mathbf{2}, 0.50 \mathrm{~mm} \times 0.40 \mathrm{~mm} \times$ $0.10 \mathrm{~mm}$ 的化合物 3 和 $0.20 \mathrm{~mm} \times 0.20 \mathrm{~mm} \times 0.20 \mathrm{~mm}$ 的化合物 4单晶, 室温下利用Rigaku Mercury 70衍射 仪或Rigaku Saturn 724+小分子衍射仪收集单晶的衍 射数据, 结果如图 S1, S2 所示. 数据的还原和结构解 析工作分别使用BrukerSHELXTL和 SHELXTL-2014 程序完成. 采用直接法确定金属原子位置, 然后用差 值函数法和最小二乘法求出全部非氢原子坐标, 最 后用最小二乘法对结构进行修正. 最终的晶体数据
利用PLATON程序进行SQUEEZE处理, 去掉了孔道 中无序的溶剂分子. 化合物的氢原子是理论加氢. 化 合物1 4的CCDC号分别为 1812535 1812537, 1812539 . 1 4 的晶体学参数列于表 1. 1 4 的结构图均用 Diamond 3.1画出. 化合物 1 4的热失重曲线和红外谱 图结果见图S3, S4.

\section{2 结果与讨论}

\section{1 化合物1的晶体结构}

$\mathrm{X}$ 射线单晶结构分析表明化合物 $\mathbf{1}$ 属于正交 $P 2_{1} 2_{1} 2_{1}$ 空间群, 它的不对称结构单元包括 2 个独立的 $\mathrm{Zn}(\mathrm{II})$ 原子中心和4个Hebimc配体. 如图2(a)所示, Zn 原子中心采取四面体配位构型, 其中 2 个 $\mathrm{O}$ 原子来自 于 2 个不同Hebimc配体, 另 2 个 $\mathrm{N}$ 原子则来自于另 2 个 不同的Hebimc配体. Hebimc配体利用羧基上 1 个 $\mathrm{O}$ 原 子和咪唑上的 1 个 $\mathrm{N}$ 原子, 以桥联的形式连接 2 个 $\mathrm{Zn}$ 原 子形成 $\mathrm{Zn}_{2}(\mathrm{Hebimc})_{2}$ 单元. 相邻的 $\mathrm{Zn}_{2}(\text { Hebimc })_{2}$ 单元通 过共享 $Z n$ 原子的方式首尾相接, 形成一维链状结构. 需要指出的是, Hebimc配体上的咪唑 $\mathrm{N}$ 原子没有脱质 子, 不参与配位, 表现出-1价. 这些Hebimc配体之间 存在强的氢键相互作用, $\mathrm{N}-\mathrm{H} \cdots \mathrm{O}$ 键的距离为 2.754 和 $2.895 \AA$ (表 $\mathrm{S} 1$ ), 链与链之间通过氢键相互作用进而 形成复杂的三维超分子网络(图2(b)).

\section{2 化合物 2 和 3 的晶体结构}

化合物 $\mathbf{2}$ 以 BDC作辅助配体合成. 化合物 $\mathbf{2}$ 结晶 于单斜 $P{ }_{2} / c$ 空间群, 不对称结构单元包括 2 个独立的 $\mathrm{Zn}(\mathrm{II})$ 中心, 2 个Hebimc配体和 1 个BDC配体. Zn 原子 中心也全部采取四面体配位模式, 其中 2 个 $\mathrm{O}$ 原子来 自 2 个 $\mathrm{BDC}$ 配体的羧基, 另外的 1 个 $\mathrm{O}$ 和 1 个 $\mathrm{N}$ 原子则 分别来自 2 个Hebimc配体. 两个Hebimc配体采取相 同的鳌合配位模式，和 2 个 $\mathrm{Zn}$ 原子形成与化合物 1 中 相同的 $\mathrm{Zn}_{2}(\text { Hebimc })_{2}$ 单元. 不同的是, 该单元被BDC 配体连接形成了 $2 \mathrm{D}$ 层状结构 (图 3(a)). 每个 $\mathrm{Zn}_{2}$ (Hebimc) $)_{2}$ 单元可以视为 $4-$ 连接节点, 每个 BDC作 为另一种 4 -连接节点, 2 可以简化为 $4-$ 连接, 且具有 $\mathbf{s q l}$ 拓扑的 $2 \mathrm{D}$ 网络结构(图3(c)); 层与层之间没有明显 的相互作用, 以 $\mathrm{ABAB}$ 的方式形成三维堆积结构 (图3(e)).

用 2,6-菜二酸 (NDC) 代替BDC时得到化合物 3 . 与化合物 $\mathbf{2}$ 相比, 化合物 $\mathbf{3}$ 的对称性稍低, 结晶于三斜 


\section{表 1 化合物 1 4 的晶体学数据}

Table 1 The crystal data for compounds 1-4

\begin{tabular}{|c|c|c|c|c|}
\hline 化合物 & 1 & 2 & 3 & 4 \\
\hline 分子式 & $\mathrm{C}_{40} \mathrm{H}_{36} \mathrm{~N}_{8} \mathrm{O}_{12} \mathrm{Zn}_{2}$ & $\mathrm{C}_{28} \mathrm{H}_{20} \mathrm{~N}_{4} \mathrm{O}_{10} \mathrm{Zn}_{2}$ & $\mathrm{C}_{16} \mathrm{O}_{5} \mathrm{~N}_{2} \mathrm{ZnH}_{11}$ & $\mathrm{C}_{34} \mathrm{H}_{29} \mathrm{~N}_{6} \mathrm{O}_{14} \mathrm{Zn}_{4}$ \\
\hline 分子量 & 948.48 & 703.22 & 376.64 & 1007.11 \\
\hline 晶系 & 正交 & 单斜 & 三斜 & 单斜 \\
\hline$a(\AA)$ & $9.1786(3)$ & $11.175(7)$ & $8.6760(5)$ & $44.664(12)$ \\
\hline$b(\AA)$ & $17.4887(6)$ & $20.450(13)$ & $11.2499(5)$ & $14.333(4)$ \\
\hline$c(\AA)$ & $27.0913(11)$ & $15.693(11)$ & $11.2174(8)$ & $25.476(7)$ \\
\hline$\alpha\left(\left(^{\circ}\right)\right.$ & 90.00 & 90 & $89.212(12)$ & 90 \\
\hline$\beta\left(^{\circ}\right)$ & 90.00 & $104.243(14)$ & $77.723(11)$ & $101.678(5)$ \\
\hline$\gamma\left({ }^{\circ}\right)$ & 90.00 & 90 & $76.930(9)$ & 90 \\
\hline$V\left(\AA^{3}\right)$ & $4348.7(3)$ & $3476(4)$ & $1041.43(12)$ & 15971(8) \\
\hline 温度(K) & $293(2)$ & $293(2)$ & $293(2)$ & $293(2)$ \\
\hline 空间群 & $P 2{ }_{1}{ }_{1} 2_{1}$ & $P 2_{1} / c$ & $P-1$ & $C 2 / c$ \\
\hline$Z$ & 4 & 4 & 2 & 8 \\
\hline 收集数据 & 11500 & 40033 & 11570 & 63995 \\
\hline 独立衍射 & 6900 & 7961 & 4760 & 17961 \\
\hline$R_{\mathrm{int}}$ & 0.0351 & 0.1023 & 0.0226 & 0.0625 \\
\hline$R_{1}(I>2 \sigma(I))$ & 0.0757 & 0.0832 & 0.0380 & 0.0586 \\
\hline $\mathrm{w} R\left(F^{2}\right)(I>2 \sigma(I))$ & 0.1130 & 0.2058 & 0.1004 & 0.1797 \\
\hline$F^{2}$ & 1.000 & 1.102 & 1.085 & 0.697 \\
\hline
\end{tabular}

$R_{1}=S\left|F_{\mathrm{o}}\right|-\left|F_{\mathrm{c}}\right| / S\left|F_{\mathrm{o}}\right|, \mathrm{wR}_{2}=\left\{S\left[w\left(\bar{F}_{\mathrm{o}}{ }^{2}-\bar{F}_{\mathrm{c}}{ }^{2}\right)^{2}\right] / \Sigma\left[w\left(\bar{F}_{\mathrm{o}}{ }^{2}\right)^{2}\right]\right\}^{1 / 2} ;\left[F_{\mathrm{o}}>4 s\left(F_{\mathrm{o}}\right)\right]$. 基于所有反射数据

(a)

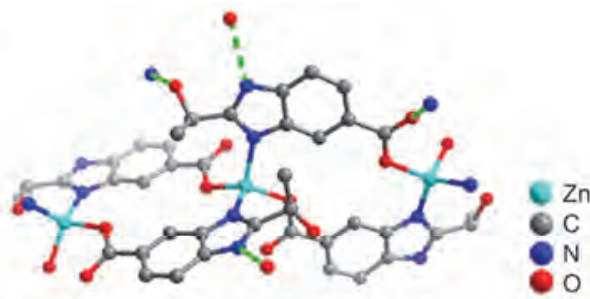

(b)

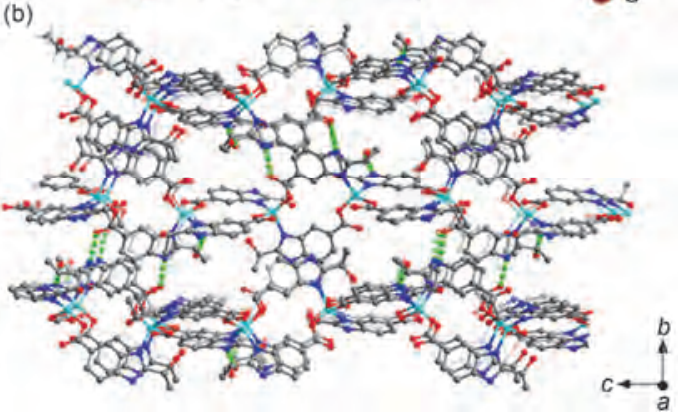

图 2 (网络版彩色) 化合物 $\mathbf{1}$ 的结构示意图. (a) 化合物 $\mathbf{1}$ 中的配位环 境图; (b) 化合物 1 通过氢键链接形成三维超分子结构的示意图

Figure 2 (Color online) The structure of 1 (a) The coordination environment in $\mathbf{1}$ with hydrogen atoms omitted for clarity; (b) the 3D supramolecular framework connected by adjacent chains through $\mathrm{N}-\mathrm{H} \cdots \mathrm{O}$ hydrogen bonds (dashed lines) in $\mathbf{1}$

$P-1$ 空间群. 但是, 在化合物 $\mathbf{3}$ 的不对称单元中, 金属 原子的配位模式以及两种配体的连接方式都与化合
物 2 非常相似。Hebimc 配体和 Zn 原子中心形成的 $\mathrm{Zn}_{2}$ (Hebimc) $)_{2}$ 单元, 在NDC配体的连接下也形成类似 的 $2 \mathrm{D}$ 层(图3(b))，层与层之间没有明显的相互作用 (图3(d)), 只是以AA的方式形成3D堆积结构(图3(f)).

\section{3 化合物 4 的晶体结构}

在层状结构的基础上, 用DOBDC代替 BDC时, 成功合成了一例具有 $3 \mathrm{D}$ 柱层式结构的化合物 4 . 化合 物 4 的不对称结构单元包括 4 个独立的 $\mathrm{Zn}$ (II) 原子中 心, 3 个ebimc配体, 1/2个 2,5-DOBDC配体, 1 个 $\mathrm{H}_{2} \mathrm{O}$ 分 子以及 1 个原位产生的 $\mathrm{OH}^{-} . \mathrm{Zn} 1$ 和 Zn3 具有相似的配 位环境, 分别与来自 ebimc配体的 1 个 $\mathrm{N}$ 原子和 2 个羧 基 $\mathrm{O}$ 原子以及 1 个原位生成的羟基 $\mathrm{O}$ 原子形成四面体 配位; $\mathrm{Zn} 2$ 与来自 2 个 ebimc配体的 $\mathrm{N}$ 原子以及分别来 自 ebimc配体和DOBDC配体的 2 个 $\mathrm{O}$ 原子同样也形成 了四面体配位. Zn4 与来自 2 个 ebimc配体的 2 个 $\mathrm{N}$ 原子 和 2 个 $\mathrm{O}$ 原子鳌和配位, 此外还与 1 个水分子配位形成 扭曲的五配位三角双雉模式.

根据连接方式的不同，这些 $\mathrm{Zn}$ 原子可以分为 3 类: (1) $\mathrm{Zn} 1$ 和 $\mathrm{Zn} 2$ 被 2 个 $\mathrm{ebmic}$ 配体鳌合形成 $\mathrm{Zn}_{2}(\mathrm{ebimc})_{2}$ 单元(图4(a));(2) 2 个 $\mathrm{Zn} 3$ 和 2 个ebmic配体 
(a)

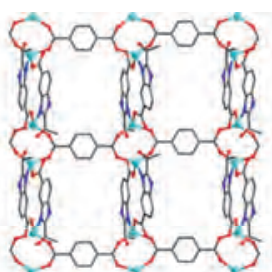

(c)

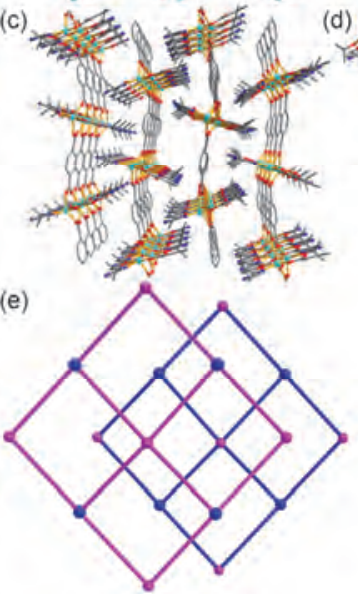

(d) (b)
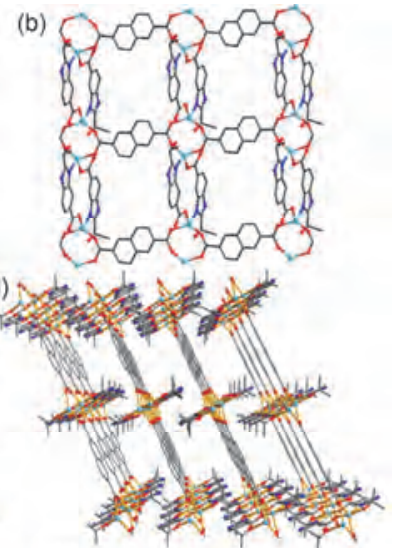

(f)

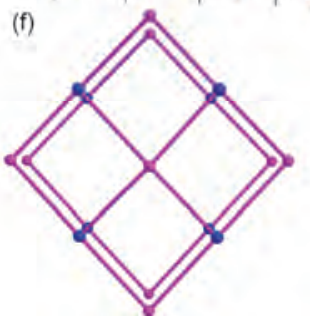

图 3 (网络版彩色)化合物 $\mathbf{2}$ 和 $\mathbf{3}$ 的结构示意图. 化合物 $\mathbf{2}$ (a) 和化合 物 3(b)的结构图; 化合物 2(c) 和化合物 3(d)的堆积图; 化合物 2(e)和 化合物 3(f)的拓扑堆积图

Figure 3 (Color online) The structure charts of 2 and 3. The 2D layer in 2 (a) and 3 (b). The staking structures of 2 (c) and $\mathbf{3}$ (d). The simplitied topology of 3D staking structure of $\mathbf{2}$ (e) and $\mathbf{3}$ (f)

形成第2种 $\mathrm{Zn}_{2}(\mathrm{ebimc})_{2}$ 单元(图4(b)); (3) $\mathrm{Zn} 4$ 则在 2 个 ebimc配体的咪唑 $\mathrm{N}$ 原子和羟基同时螯合配位作用下, 以单核的形式存在 (图4(c)). $\mathrm{Zn}_{2}$ (ebimc) $)_{2}$ 单元和单核 $\mathrm{Zn}$ 之间相互连接形成2D层结构(图4(e), (f)). DOBDC 连接邻近的层形成3D柱层式框架(图 $4(\mathrm{~g})$ ), 通过拓扑 分析对这个复杂的 $3 \mathrm{D}$ 结构进行简化. Zn1 和 Zn2所在 的 $\mathrm{Zn}_{2}$ (ebimc $)_{2}$ 单元可以简化为 5 -连接的节点, $\mathrm{Zn} 3$ 所 代表的 $\mathrm{Zn}_{2}(\mathrm{ebimc})_{2}$ 单元简化为 4-连接节点, $\mathrm{Zn} 4$ 和 DOBDC都可以看作是桥联的配体 (图4(d)), 化合物 4 可以简化为 $(4,5)$ - 连接的 $3 \mathrm{D}$ 网络, 其点群符号是 $\left(4^{2} \cdot 5^{3} \cdot 6^{5}\right) 2\left(5^{2} \cdot 6^{4}\right)($ 图 $4(\mathrm{~h}))$.

值得注意的是, 化合物 $\mathbf{1}$ 中 $\mathrm{Zn}_{2}(\mathrm{Hebimc})_{2}$ 首尾相接 形成 $1 \mathrm{D}$ 链, 引人辅助二羧酸配体后, $\mathrm{Zn}_{2}(\mathrm{Hebimc})_{2} \mathrm{SBU}$ 被羧酸连接形成 $2 \mathrm{D}$ 层状结构的化合物 2 和 3 . 在化合 物 1 3 中, Hebimc配体咪唑环上的氮没有脱去质子, 而在化合物 4 中, 加人三苯基膦作为有机碱, 使得 Hebimc配体上的羧基和咪唑基同时脱去质子, 表现 出高的配位能力. 咪唑氮原子和羟基氧原子以鳌合 的形式又捕获了 1 个单核的锌原子. $\mathrm{Zn}_{2}(\mathrm{ebimc})_{2}$ 单元 和单核锌通过ebimc配体形成二维层状结构, 再通过

(a)

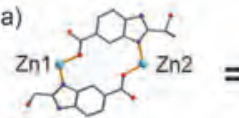

(c)

(e)

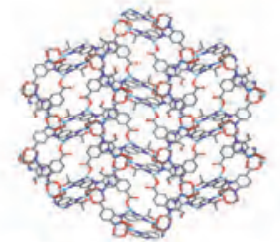

(g)

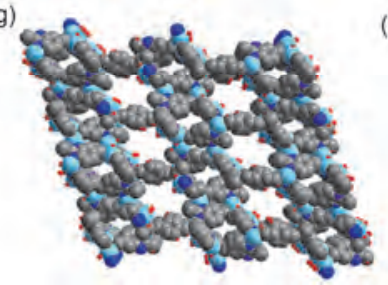

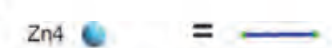

(b)

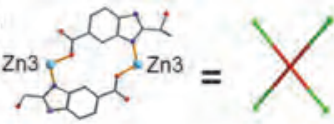

(d)

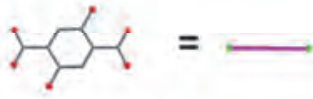

(f)

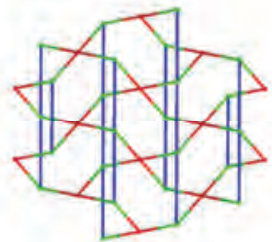

(h)

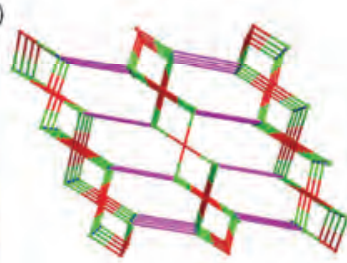

图 4 (网络版彩色)化合物 $\mathbf{4}$ 的结构示意图. (a) 一类 $\mathrm{Zn}_{2}(\mathrm{Hebimc})_{2}$ SBUs简化成 5-链接节点; (b) 另一类 $\mathrm{Zn}_{2}(\mathrm{Hebimc})_{2} \mathrm{SBUs}$ 简化成 4-链接 节点; (c) $\mathrm{ZN}_{4}$ 简化成键; (d) DOBDC配体简化成键; (e) 配合物 4 中 $\mathrm{Zn}_{2}$ (Hebimc) $)_{2} \mathrm{SBUs}$ 与 $\mathrm{ZN}_{4}$ 形成 $2 \mathrm{D}$ 层; (f) 配合物 4 中 $\mathrm{Zn}_{2}(\text { Hebimc })_{2}$ SBUs 与 $\mathrm{ZN}_{4}$ 形成 $2 \mathrm{D}$ 层拓扑图; (g) 配合物 $\mathbf{4}$ 的 $3 \mathrm{D}$ 结构图; (h) 配合物 4 的 3D网络拓扑图

Figure 4 (Color online) The structure charts of 4. (a) One kind of $\mathrm{Zn}_{2}(\text { Hebimc) })_{2} \mathrm{SBUs}$ considered as the 5-connected nodes; (b) the other kind of $\mathrm{Zn}_{2}(\mathrm{Hebimc})_{2} \mathrm{SBUs}$ considered as the 4-connected nodes; DOBDC ligands (c) and $\mathrm{ZN}_{4}$ (d) considered as the 2-connected bonds; (e) the $2 \mathrm{D}$ layer formed by $\mathrm{Zn}_{2}(\text { Hebimc })_{2} \mathrm{SBUs}$ and $\mathrm{ZN}_{4}$; (f) the $2 \mathrm{D}$ simplified layer formed by $\mathrm{Zn}_{2}(\text { Hebimc })_{2} \mathrm{SBUs}$ and $\mathrm{ZN}_{4}$; (g) the $3 \mathrm{D}$ framework of 4 ; (h) the simplified topology of 4

辅助配体DOBDC的桥联形成了3D柱层式框架结构. 因此, 利用辅助配体和有机碱, 可以逐步实现从 $1 \mathrm{D}$ 到3D MOFs材料的结构调控.

\section{4 化合物4负载Ag纳米粒子及光催化性能}

通常由外部还原剂制备的银纳米颗粒 (Ag NPs) 易导致聚集体的形成，在温和条件下制备均匀分散 的小尺寸 Ag NPs仍然是一个挑战 ${ }^{[22 ~ 27]}$. 化合物 4 具有 $3 \mathrm{D}$ 框架和较小的孔道尺寸，因此我们考虑利用化合 物 4来合成均匀分散的 $\mathrm{Ag} N \mathrm{NPs}$. 将化合物 $4(120 \mathrm{mg})$ 浸人 $\mathrm{AgNO}_{3}(10 \mathrm{mmol} / \mathrm{L}, 10 \mathrm{~mL})$ 的水溶液中, 在室温 下避光保存 $1 \mathrm{~d}$, 淡黄色晶体逐渐变成灰色. 透射电 子显微镜(TEM)图像表明在晶体中形成了 $\mathrm{Ag} \mathrm{NPs}$ (图 $5(\mathrm{~b})$ ), 这些Ag NPs分散均匀、尺寸均一(约 $5 \mathrm{~nm}$ ), 晶 格条纹间距为 $0.23 \mathrm{~nm}$, 与 $\mathrm{Ag}(111)$ 的 $d$ 值接近(图 5(d)). 

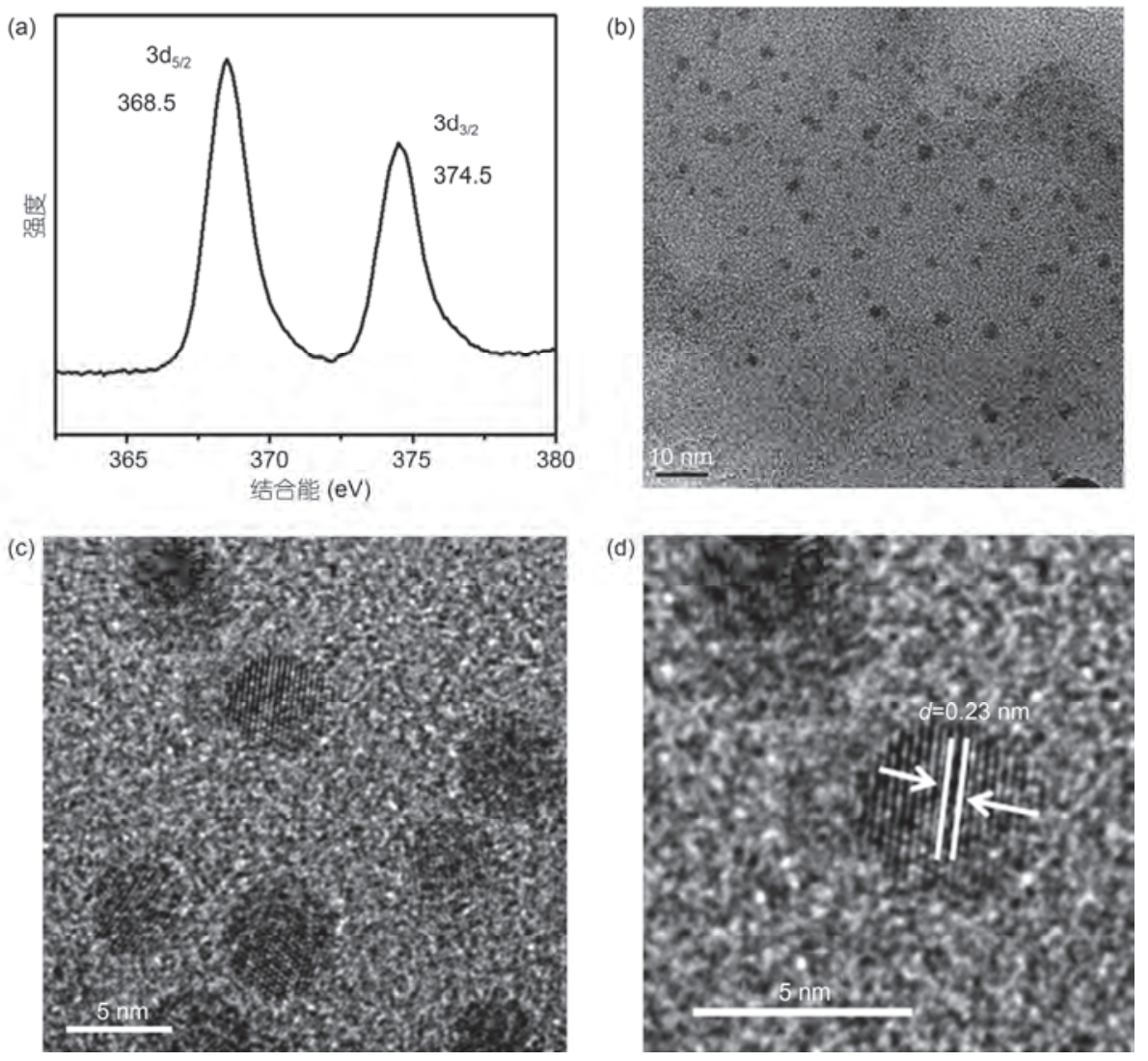

图 5 (网络版彩色)Ag@4 的XPS谱图(a), TEM图像(b)和HRTEM图像((c), (d))

Figure 5 (Color online) The XPS spectra (a), the typical TEM (b) and HRTEM ((c), (d)) of Ag@4

负载后的样品等离子体发射光谱(ICP-AES)结果表 明, Ag NPs 在化合物 4 中的重量百分比为 $3.47 \%$. 此 外, $X$ 射线光电子能谱(XPS)表征结果显示 $\mathrm{Ag} @ 4$ 在 368.5 和 $374.5 \mathrm{eV}$ 分别出现了 $3 \mathrm{~d}_{5 / 2}$ 和 $3 \mathrm{~d}_{3 / 2}$ 峰, 表明 $\mathrm{Ag}(0)$ 的存在(图5(a)). $\mathrm{Ag} @ 4$ 的X射线能谱(EDX)表征 结果表明, $\operatorname{Ag}(0)$ 和 $Z n(I I)$ 共存于固体中(图S5). X射线 粉末衍射(PXRD)结果也表明化合物 4在负载 Ag NP 后, 主体框架依然稳定存在(图S4). 以上结果说明化 合物 4 成功实现了温和条件下对 $\mathrm{Ag}$ NPs的负载.

利用其在可见光照下对结晶紫 $(\mathrm{CV})$ 水溶液的光 降解实验, 评估了均匀分散的银纳米粒子复合材料 $\mathrm{Ag} @ \mathbf{4}$ 的光催化活性. 在室温下, 将 $20 \mathrm{mg} \mathrm{Ag} @ 4$ 加人 $10 \mathrm{~mL}, 50 \mathrm{mg} / \mathrm{L}$ 的 CV 水溶液中, 在暗处搅拌 $30 \mathrm{~min}$ 后, 置于 $500 \mathrm{~W}$ 氙灯下用可见光照射, 光源和反应溶 液之间的距离是 $15 \mathrm{~cm}$, 每隔 $20 \mathrm{~min}$ 取出 $2 \mathrm{~mL}$ 溶液, 直到结晶紫溶液变成无色, 取出的溶液过滤后依次

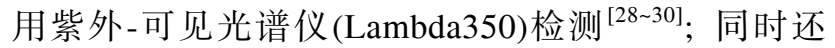

进行了未加光催化剂的空白实验(图S6). 通过 CV 在 $580 \mathrm{~nm}$ 处的紫外可见特征吸收峰, 来检测其光降解 程度, 结果如图 S7所示. CV 通过 $\mathrm{Ag} @ 4$ 的光降解作用 逐渐降解, 在 $80 \mathrm{~min}$ 内, 其紫外可见吸收峰逐渐变弱 直至消失, 表明 $\mathrm{Ag} @$ 4具有较好光催化活性. PXRD结 果显示反应后复合材料 $\mathrm{Ag} @ 4$ 的骨架几乎保持不变, 表明其稳定性良好(图S2).

\section{3 结论}

本文利用咪唑衍生物Hebimc作为配体和金属锌 盐反应, 制备了一种新的次级构筑单元 $\mathrm{Zn}_{2}(\mathrm{ebimc})_{2}$. 基于此单元进行了一系列实验，成功实现了从一维到 三维的结构调控. 由于功能化的-OH基团, 化合物 $\mathbf{4}$ 在 温和的室内条件下即可产生尺寸均一、分散均匀的 $\mathrm{Ag}$ NPs复合物 $(A g @ 4)$, 得到的 $A g @ 4$ 具有不错的光催化 降解 CV 性能. 这些结果为构建具有丰富拓扑网络及 不同取代基团的新型功能性MOF材料提供了新思路. 


\section{参考文献}

1 Buru C T, Li P, Mehdi B L, et al. Adsorption of a catalytically accessible polyoxometalate in a mesoporous channel-type metal-organic Framework. Chem Mater, 2017, 29: 5174-5181

2 Tang Y H, Wang F, Liu J X, et al. Diverse tetrahedral tetrazolate frameworks with N-rich surface. Chem Comm, 2016, 52: 5625-5628

3 Zhang H X, Liu M, Wen T, et al. Synthetic design of functional boron imidazolate frameworks. Coord Chem Rev, 2016, 307: 255-266

4 Sung C H, Deng H, Miyasaka K, et al. Extra adsorption and adsorbate superlattice formation in metal-organic frameworks. Nature, 2015, 527: 503-507

5 Lin Q, Wu T, Zheng S T, et al. A chiral tetragonal magnesium-carboxylate framework with nanotubular channels. Chem Comm, 2011, 47: 11852-11854

6 Zhou H C, Long J R, Yaghi O M. Introduction to metal-organic frameworks. Chem Rev, 2012, 112: 673-674

7 Yan Y, Kolokolov D I, Da S I, et al. Porous Metal-organic polyhedral frameworks with optimal molecular dynamics and pore geometry for methane storage. J Am Chem Soc, 2017, 139: 13349-13360

8 Morris R E, Brammer L. Coordination change, lability and hemilability in metal-organic frameworks. Chem Soc Rev, 2017, 46: 5444-5462

9 Chui S S Y, Lo S M F, Charmant J P H, et al. A chemically functionalizable nanoporous material $\left[\mathrm{Cu}_{3}\left(\mathrm{TMA}_{2}\left(\mathrm{H}_{2} \mathrm{O}\right)_{3}\right]_{n}\right.$. Science, 1999, 283: 1148-1150

10 Latroche M, Surblé S, Serre C, et al. Hydrogen storage in the giant-pore metal-organic frameworks MIL-100 and MIL-101. Angew Chem Int Ed, 2006, 45: 8227-8231

11 Chae H K, Siberio-Pérez D Y, Kim J, et al. A route to high surface area, porosity and inclusion of large molecules in crystals. Nature, 2004, 427: 523-527

12 Kandiah M, Nilsen M H, Usseglio S, et al. Synthesis and stability of tagged UiO-66 Zr-MOFs. Chem Mater, 2010, 22: 6632-6640

13 Doonan C J, Morris W, Furukawa H, et al. Isoreticular metalation of metal-organic frameworks. J Am Chem Soc, 2009, 131: $9492-9493$

14 Tu B, Pang Q, Ning E, et al. Heterogeneity within a mesoporous metal-organic framework with three distinct metal-containing building units. J Am Chem Soc, 2015, 137: 13456-13459

15 Bai Y L, Tao J, Huang R B, et al. The designed assembly of augmented diamond networks from predetermined pentanuclear tetrahedral units. Angew Chem Int Ed, 2008, 47: 5344-5347

16 Wang F, Kusaka S, Hijikata Y, et al. Development of a porous coordination polymer with a high gas capacity using a thiophene-based bent tetracarboxylate ligand. ACS Appl Mater Interfaces, 2017, 9: 33455-33460

17 Fujiwara Y, Kadota K, Nagarkar S S, et al. Synthesis of oligodiacetylene derivatives from flexible porous coordination frameworks. J Am Chem Soc, 2017, 139: 13876-13881

18 Duan J, Higuchi M, Zheng J, et al. Density gradation of open metal sites in the mesospace of porous coordination polymers. J Am Chem Soc, 2017, 139: 11576-11583

19 Sun F, Yin Z, Wang Q Q, et al. Tandem postsynthetic modification of a metal-organic framework by thermal elimination and subsequent bromination: Effects on absorption properties and photoluminescence. Angew Chem Int Ed, 2013, 52: 4538-4543

20 Wang L, Yang E, Kang Y, et al. Synthesis and photoluminescent properties of four homochiral supramolecular compounds with butterflylike chains. Inorg Chem Commun, 2014, 46: 219-222

21 Tang Y H, Wu X, Wang F, et al. Sythesis of zeolite-like metal-organic frameworks from a dual-ligand strategy. CrystEngComm, 2017, 19: 2549-2552

22 Zhang H X, Liu M, Bu X, et al. Zeolitic BIF crystal directly producing noble-metal nanoparticles in its pores for catalysis. Sci Rep, 2014, 4: 3923-3928

23 Liu M, Zhang D X, Chen S, et al. Loading Ag nanoparticles on Cd(II) boron imidazolate framework for photocatalysis. J Solid State Chem, 2016, 237: 32-35

24 Liu M, Chen S, Wen T, et al. Encapsulation of $\operatorname{Ln}(\mathrm{III})$ ions/Ag nanoparticles within Cd(II) boron imidazolate frameworks for tuning luminescence emission. Chem Comm, 2016, 52: 8577-8580

25 Khan I A, Qian Y, Badshah A, et al. Highly porous carbon derived from MOF-5 as a support of ORR electrocatalysts for fuel cells. ACS Appl Mater Interfaces, 2016, 8: 17268-17275

26 Whitford C L, Stephenson C J, Gómezgualdrón D A, et al. Elucidating the nanoparticle-metal organic framework interface of Pt@ZIF-8 catalysts. J Phys Chem C, 2017, 121: 25079-25091

27 Gole B, Sanyal U, Banerjee R, et al. High loading of Pd nanoparticles by interior functionalization of MOFs for heterogeneous catalysis. Inorg Chem, 2016, 55: 2345-2354

28 Zhang B, Feng M L, Li J, et al. Syntheses, crystal structures, optical and photocatalytic properties of four small-amine-molecule-directed M-Sn-Q (M=Zn, Ag; Q=S, Se) compounds. Cryst Growth Des, 2017, 17: 1235-1244

29 Liu J, Wang F, Liu L Y, et al. Interpenetrated three-dimensional copper-iodine cluster-based framework with enantiopure porphyrin- like templates. Inorg Chem, 2016, 55: 1358-1360

30 Lei X W, Yue C Y, Feng L J, et al. Syntheses, crystal structures and photocatalytic properties of four hybrid iodoargentates with zeroand two-dimensional structures. CrystEngComm, 2016, 18: 427-436 


\title{
Synthesis and properties of metal organic frameworks with novel metal-imidazolate secondary building units
}

\author{
Yuhuan Tang ${ }^{1,2}$, Fei Wang ${ }^{1 *} \&$ Jian Zhang ${ }^{1 *}$ \\ ${ }^{1}$ State Key Laboratory of Structural Chemistry, Fujian Institute of Research on the Structure of Matter, Chinese Academy of Sciences, Fuzhou \\ 350002, China; \\ ${ }^{2}$ University of Chinese Academy of Sciences, Beijing 100049, China \\ * Corresponding authors, E-mail: wangfei04@fjirsm.ac.cn; zhj@fjirsm.ac.cn
}

Metal-organic frameworks (MOFs) are a class of inorganic-organic hybrid materials and have potential applications in many fields such as gas adsorption and storage, catalysis, ion exchange and separation, chemical sensing and so on. It has attracted a great deal of chemists' attention. Moreover, the development of new secondary building units (SBUs) is of great importance for the synthesis of MOFs. In this work, we choose 2-(1-hydroxy-ethyl)-3H-benzoimidazole-5-carboxylic acid (Hebimc) as organic ligand, which is a trifunctional imidazolate derivative comprising metal coordination groups and diverse functional sites. In this ligand, the imidazolate unit can provide $\mu 2$-bridging coordination mode to form zeolitic MOFs, the hydroxy unit could offer active site for further reactions, the benzene ring may offer $\pi-\pi$ interaction with guest molecules for fascinating structures, and the carboxy unit also could coordinate to metal center. So far, MOFs assembled by Hebimc ligand and auxiliary dicarboxylic ligands have not been reported yet.

By employing the Hebimc ligand and introduce auxiliary dicarboxylic ligands to assemble with different transition metal salts, respectively, four MOFs from one-dimensional (1D) to three-dimensional (3D), namely, $\mathrm{Zn}_{2}(\text { Hebimc })_{4}(\mathbf{1}), \mathrm{Zn}_{2}(\text { Hebimc })_{2}(\mathrm{BDC}) \cdot x \mathrm{G}(\mathbf{2}, \mathrm{BDC}=$ terephthalic acid, $\mathrm{G}=$ guest molecule $), \mathrm{Zn}_{2}(\text { Hebimc })_{2}(\mathrm{NDC}) \cdot x \mathrm{G}$ (3, NDC=2,6-naphthalene diacid), $\mathrm{Zn}_{8}(\mathrm{Hebimc})_{6}(\mathrm{DOBDC})(\mathrm{OH})_{2}\left(\mathrm{H}_{2} \mathrm{O}\right)_{2} \cdot x \mathrm{G}(4, \mathrm{DOBDC}=2,5$-dih-ydroxyterephthalic acid), have been synthesized under different reaction conditions. 1 was synthesized by the reaction of Hebimc and $\mathrm{ZnSiF}_{6} \cdot 6 \mathrm{H}_{2} \mathrm{O}$ in $\mathrm{H}_{2} \mathrm{O}$ at $100^{\circ} \mathrm{C}$ for $3 \mathrm{~d}$ (yield: $40 \%$ based on Hebimc); 2 was prepared by the reaction of Hebimc and $\mathrm{BDC}$ and $\mathrm{ZnSiF}_{6} \cdot 6 \mathrm{H}_{2} \mathrm{O}$ in isopropanol/water $(6: 3, \mathrm{v}: \mathrm{v})$ at $100^{\circ} \mathrm{C}$ for $3 \mathrm{~d}$ (yield: $65 \%$ based on Hebimc); 3 was prepared by the reaction of Hebimc, NDC, pyrazine, tetraethylammonium bromide (TEAB) and $\mathrm{Zn}\left(\mathrm{NO}_{3}\right)_{2} \cdot 6 \mathrm{H}_{2} \mathrm{O}$ in $N, N$-dimethyl formamide (DMF)/water $(3: 1.5, \mathrm{v}: \mathrm{v})$ at $120^{\circ} \mathrm{C}$ for $3 \mathrm{~d}$ (yield: $78 \%$ based on Hebimc); 4 was prepared by the reaction of Hebimc, DOBDC, pyrazine, triphenylphosphine and $\mathrm{Zn}\left(\mathrm{NO}_{3}\right)_{2} \cdot 6 \mathrm{H}_{2} \mathrm{O}$ in DMF/water $(6: 1.5, \mathrm{v}: \mathrm{v})$ at $120^{\circ} \mathrm{C}$ for $3 \mathrm{~d}$ (yield: $50 \%$ based on Hebimc).

Single crystal X-ray diffraction was used to characterize their structures. It is noted that Hebimc ligands in this structures are chelate coordinate to metal centers to form $\mathrm{Zn}_{2}(\text { Hebimc })_{2}$ SBUs, which is unusual in MOFs. 1 crystallized in orthorhombic $P 22_{1} 2_{1} 2_{1}$ space group and exhibited 1D chain connected by N-H...O hydrogen bonds into a supermolecular structure. By introducing auxiliary dicarboxylic ligands, two 2D layers $\mathbf{2}$ and $\mathbf{3}$ are obtained. 2 crystallized in monoclinic $P 2_{1} / c$ space group and exhibited $2 \mathrm{D}(4,4)$-sql layer. There is no obvious interaction between the layers, packing a 3D accumulation structure in the ABAB way. 3 crystallized in triclinic P-1 space group and exhibited an 2D (4,4)-sql layer, packing a 3D accumulation structure in the AA way. Under the inducement of the ligand DOBDC, a pillar-layer 3D structure 4 is obtained. 4 crystallized in monoclinic $C 2 / c$ space group and can be simplified as a (4,5)-connected network with the point symbol $\left(4^{2} \cdot 5^{3} \cdot 6^{5}\right) 2\left(5^{2} \cdot 6^{4}\right)$. Besides, hydroxy-functional neutral compound 4 shows an obvious degradation effect on photocatalysis of crystal violet aquous after loading $\mathrm{Ag}$ nanoparticles (NPs). These results demonstrated that the mixed ligand approach is a successful route for the construction of MOFs.

metal-organic framework, crystal engineering, controllable synthesis, single crystal structure, dye degradation doi: 10.1360/N972018-00062 